\title{
Synthesis and Characterization of $\mathrm{CNT} / \mathrm{TiO}_{2}$ Composites Thermally Derived from MWCNT and Titanium(IV) $n$-Butoxide
}

\author{
Won-Chun $\mathrm{Oh}^{\circ}$ and Ming-Liang Chen \\ Department of Athanced Materials \& Science Engineering, Hanseo Lniversity, Chungnam 356-706, Korea \\ E-mail: wc ohíhanseo.ac.kr \\ Received Ōtober 17, 2007
}

\begin{abstract}
Two kinds of $\mathrm{CNT} / \mathrm{TiO}_{2}$ composite photocatalysts were sy'nthesized with multi-walled carbon nanotubes (MWCNTs) and titanium(IV) n-butoxide (TNB) by a MCPBA oxidation method. Since MWCNTs had charge transfer and semiconducting the $\mathrm{CNT/TiO}$ z composite shows a good photo-degradation activity. The $\mathrm{XRD}$ patterns reveal that only anatase phase can be identified for MCT composite. but the HMCT composite synthesized with $\mathrm{HCl}$ treatment was observed the mixed phase of anatase and nutile. The EDX spectra were shown the presence as major elements of Ti with strong peaks. From the SEM results. the sample MCT and HMCT synthesized by the thermal decomposition with TNB show a homogenous sample with only individual MWCNTs covered with $\mathrm{TiO}_{2}$ without any jam-like aggregates between $\mathrm{CNTs}$ and $\mathrm{TiO}_{2}$. From the photocatalytic results. we could be suggested that the excellent activity of the $\mathrm{CNT} / \mathrm{TiO}_{2}$ composites for organic dye and UV irradiation time could be attributed to combination effects between $\mathrm{TiO}_{2}$ and MWCNTs with plausible photodegradation mechanism.
\end{abstract}

Key Words : Carbon ninotube, Titanium(IV) $n$-butoxide, XRD. SEM. Photocatalysis

\section{Introduction}

Carbon based $\mathrm{TiO}_{2}$ composites have attracted much attention and become a very active field of research due to their unique properties and promising applications for the big problem of pollutions. . $^{1-5}$ Especially. carbon nanotubes (CNTs) have attracted considerable attention in recent years owing to their remarkable photoelectrical and mechanical properties. ${ }^{6}$ As well these composites have intrinsic properties as the materials exhibit cooperative or synergitic effects. Titania is an important semiconducting material. which has been applied as photcataly'sis and photosensitive active materials due to its excellent physical and chemical properties. ${ }^{7.8}$ One of its most important applications is to behave as photocatalyst for some chemical reaction for the water contaminated with organic pollutants such as dyes and aromatic or cyclic compounds. ${ }^{5}$ Multi-walled carbon nanotubes (MWCNTs) could be considered as a good support for materials with field emission display's and photocatalytic properties. On the other hand. a combination of $\mathrm{TiO}_{2}$ and MWCNT could create many active sites for the photocatalytic degradation. Different research techniques have already been used to coat carbon supports for the special usages. The titanium(IV) $n$-butoxide (TNB) treated carbon composite was synthesized with activated carbon fiber ${ }^{11}$ in order to obtain adsorptive materials for pollutant gas and photocataly'sis for the degradation of dy'es. ${ }^{12}$ Activated carbon materials and $\mathrm{TiO}_{2}$ composites by mechanical and hydrothermal treatment demonstrated very good performance in the photocataytic degradation of methylene blue. ${ }^{13.14}$ For the number of applications. however. the practical use of $\mathrm{TiO}_{2}$ in powder shows some drawbacks like the immobilization of $\mathrm{TiO}_{2}$ on fixed supports. which was found to reduce the functional efficiency of the material. The first trial of CNT coating on the oxide layers was studies by Seeger $e t a l^{15}$ and Hernandi $e t a{ }^{16}$ CNT are attractive and comparative catalyst supports when compared to activated carbon due to the combination of their electronic. adsorption. mechanical and thermal properties. The characteristic electronic properties of CNT are that they can be metallic as well as semiconductiong depending on their geometry. ${ }^{17}$ Composites containing CNT are believed to provided many applications and exhibit combination effects between carbon phases and metal oxides.

In this study. we present the synthesis and characterization of $\mathrm{CNT} / \mathrm{TiO}_{2}$ composites synthesized by an improved oxidation method. The structural variations. surface state and elemental compositions were investigated through preparation of two kinds of $\mathrm{CNT} / \mathrm{TiO}_{2}$ composites. X-ray diffraction (XRD). energy' dispersive X-ray (EDX) spectroscopy. scanning electron microscopy (SEM) and UV/VIS spectroscopy were characterized for these new photocatalysts.

\section{Experimental}

Materials. Crystalline MWCNT powder of 95.9 wt.\% purity from Nanokarbon (Nanokarbon Co.. Ltd. Korea) was used as a starting material. The XRD pattern for the pristine MWCNT was shown in Figure 1. Reagents (benzene and ethyl alcohol) were purchased as reagent-grade from Duksan chenical $\mathrm{Co}$ and Daejung cheimical $\mathrm{Co}$. and used without further purification unless otherwise stated. Evaporation and concentration in vacuum were done at a water aspirator pressure and compounds were dried at $1.33 \mathrm{~Pa}$. For the comparison, the $\mathrm{TiO}_{2}$ photocatalysts used were the commer- 

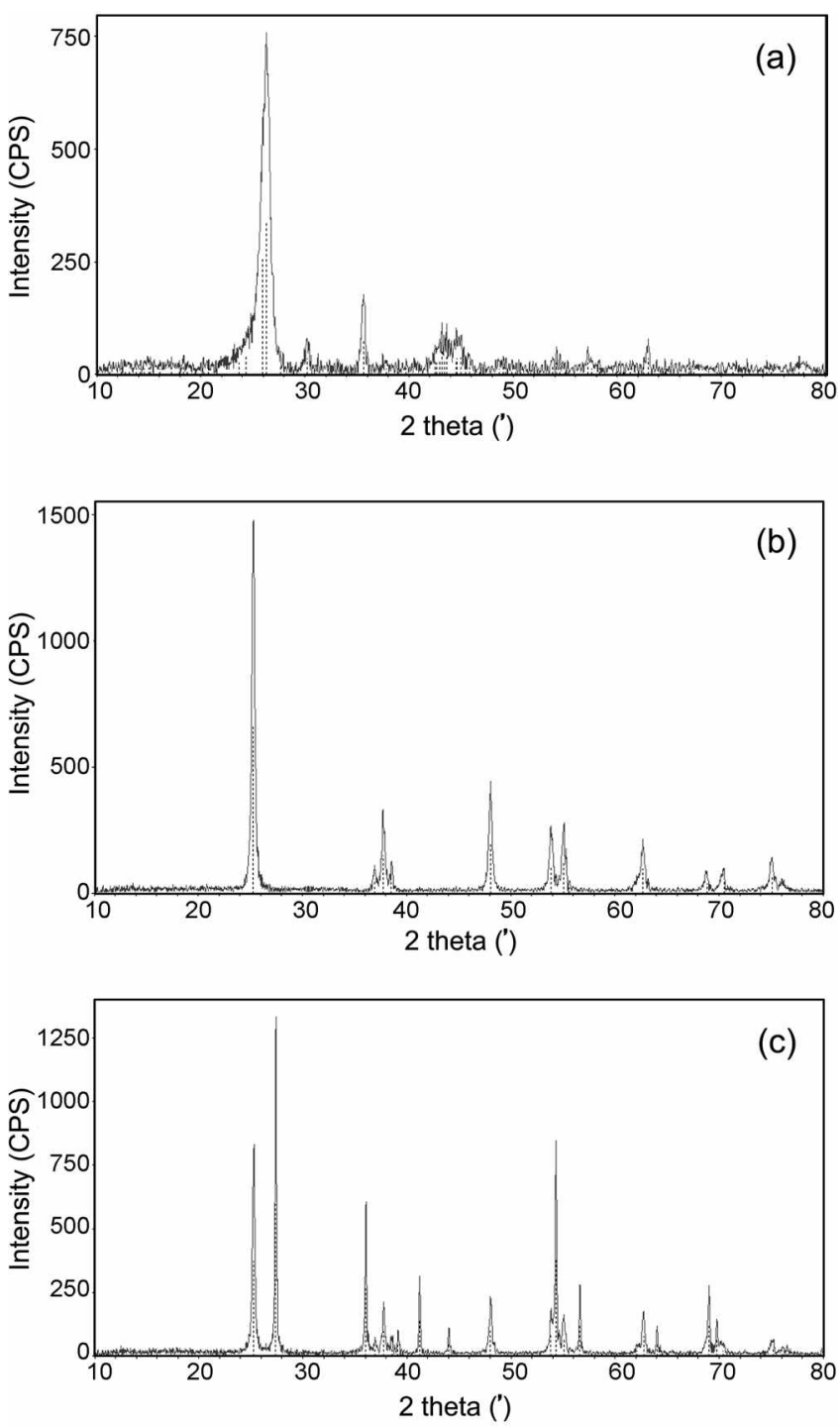

Figure 1. XRD pattens of CNT/T1O composites; (a) Pristine MWCNT, (b) MCT composite catalyst and (c) HMCT composite catalyst.

cially available (Duk-San Pure Chemical Co., Korea), which were used of a single phase of anatase with secondary particles of about $80-150 \mu \mathrm{m}$ aggregated from the primary particles of about $30-50 \mu \mathrm{m}$ with BET surface area of about $83 \mathrm{~m}^{2} / \mathrm{g}$. The titanium(IV) $n$-butoxide(TNB. $\mathrm{C}_{16} \mathrm{H}_{36} \mathrm{O}_{4} \mathrm{Ti}$ ) as a titanium source for the synthesis of $\mathrm{CNT} / \mathrm{TiO}_{2}$ composites were purchased as reagent-grade from Acros Organics, USA.

Chemical oxidation on the MWCNT surface. $m$-clloroperbenzoic acid ( $c a .0 .96 \mathrm{~g}$ ) was suspended in $60 \mathrm{~mL}$ benzene as a solvent, and MWCNT ( $c a .40 \mathrm{mg}$ ) was added, the mixture was refluxed in an air atmosphere for $6 \mathrm{~h}$. Then the solvent was dried at the boiling point $(353.13 \mathrm{~K})$ of benzene. The solid precipitates were transformed to a dark brown color. After completion. dark brown precipitates were washed with ethyl alcohol and then dried at $363 \mathrm{~K}$. Before the TNB treatment for the oxidized MWCNT. we prepared to two kinds of samples of non and $\mathrm{HCl}$ treatment to the oxidized MWCNT

Preparation of $\mathrm{CNT} / \mathrm{TiO}_{2}$ composite. We prepared two kinds of oxidized MWCNT (non and $\mathrm{HCl}$ treated) slurry solutions with pristine concentrations of $20 \mathrm{~mL}$ of TNB for the synthesis of $\mathrm{CNT} / \mathrm{TiO}_{2}$ composites. For the synthesis. $0.5 \mathrm{~g}$ of powdered two kinds of oxidized MWCNT were mixed into $20 \mathrm{~mL}$ of TNB aqueous solution and stirred for 5 hours at $333 \mathrm{~K}$. Then, the powder mixtures of TNB with two kinds of oxidized MWCNT were heated at $973 \mathrm{~K}$ for $\mathrm{l} \mathrm{h}$ for the thermal decomposition. Before heat treatment. the solvent in the mixtures was vaporized at $343 \mathrm{~K}$ for 1 hour. Finally, these samples were dried at $368 \mathrm{~K}$ for 72 hours in an air atmosphere. The summary of synthesis procedure and nomenclatures for the $\mathrm{CNT/TiO}$, composites listed in Table 1.

Characterization of $\mathrm{CNT} / \mathrm{TiO}_{2}$ composites. For the determination of the crystallographic structure of the inorganic constituent of the composites. XRD patterns were taken using an $X$-ray generator (Shimatz XD-Dl, Japan) with $\mathrm{Cu} \mathrm{K} \alpha$ radiation. EDX spectra were also used for the elemental analysis of the samples. SEM (JSM-5200 JOEL. Japan) was used to observe the surface state and structure of two kinds of $\mathrm{CNT} / \mathrm{TiO}_{2}$ composites. For the analysis of photo-degradation effects, UV-VIS spectra for the CNT/ $\mathrm{TiO}_{2}$ composites were recorded using a Genspec (Hitachi, Japan) spectrometer.

Photocatalytic effect. Photocatalytic activities were evaluated by $\mathrm{MB}$ degradation in aqueous media under ultraviolet light irradiation. For UV irradiation, the reaction tube was located axially and held in UV lamp ( 20 W. 365 $\mathrm{nm}$ ) box. The lamp was used at the distance of $100 \mathrm{~mm}$ from the solution in darkness box. The initial MB concentration (ci) was $1.0 \times 10^{-6} \mathrm{~mole} / \mathrm{L}$. The amount of suspended CNT/

Table 1. Summary of Synthesis Procedure and Nomenclatures of Samples Prepared with Non and Hydrochloric Acid Treatment to MWCNT

\begin{tabular}{|c|c|}
\hline Synthesis Procedure & Nomenclatures \\
\hline $\begin{array}{l}\text { 1) MWCNT + MCPBA ( } m \text {-chloroperbenzoic acid) : Oxidation of MWCNT surface } \\
\text { 2) 1) + TNB (Titanium } n \text {-butoxide, } 99.99 \% \text { ) } \\
\text { 3) Thernal decomposition (at } 973 \mathrm{~K} \text { ) }\end{array}$ & $\mathrm{MCT}$ \\
\hline $\begin{array}{l}\text { 1) MWCNT + MCPBA (m-chloroperbenzoic acid) : Oxidation of MWCNT surface } \\
\text { 2) l) compound + } 0.01 \mathrm{M} \mathrm{HCl} \text { treatment : Fonmation of surface group } \\
\text { 3) 2) + TNB (Titanium } n \text {-butoxide, } 99.99 \% \text { ) } \\
\text { 4) Themal decomposition (at } 973 \mathrm{~K} \text { ) }\end{array}$ & HMCT \\
\hline
\end{tabular}


$\mathrm{TiO}_{2}$ composites was kept at $0.005 \mathrm{~g} / \mathrm{mL}$. Before turning on illunination, the suspension containing $\mathrm{MB}$ and $\mathrm{CNT} / \mathrm{TiO}$ 2 composites was stirred with ultrasonicater in the dark condition for 3 minutes to establish an adsorption-desorption equilibrium. The suspension was irradiated with ultraviolet light as a function of irradiation time. Samples were then withdrawn regularly from the reactor and removal of dispersed powders through centrifuge. The clean transparent solution was analyzed by UV/NIS spectroscopy. The concentration of $\mathrm{MB}$ in the solution was determined as a function of irradiation time from the absorbance region at a UV wavelength line of $256 \mathrm{~cm}$.

\section{Results and Discussion}

Structural and elemental analysis. XRD technique was used to determine the crystallographic structure of the inorganic constituent of the composite. The components profile of carbon and $\mathrm{TiO}_{2}$ for the conposite catalysts determined by XRD was shown in Figure 1. In case of sample MCT (Fig. 1(b)) synthesized without $\mathrm{HCl}$ treatment procedure. the XRD patterns reveal that only anatase phase can be identified for $\mathrm{CNT} / \mathrm{TiO}$ : composite. But, the sample HMCT (Fig. 1(c)) synthesized with $\mathrm{HCl}$ treatment was observed the mixed phase of anatase and rutile. In the former studies for the $\mathrm{TiO}_{2}$ formation from TNB. ${ }^{18.15}$ the XRD results were shown that the anatase phase has disappeared with the increasing temperature process and the rutile phase has occurred at relatively high temperature. In this study. it was worthy of notice that the characteristic peaks of MWCNT can hardly been identified from all the patterns of composite catalysts. It is considered that the peak widths broaden slightly and gradually with a decrease with peak intensity of MWCNT for the composite catalysts. The absence of MWCNT aggregated in the composite catalysts proves a homogeneous dispersion of MWCNT in $\mathrm{TiO}_{2}$ matrix. which was also supported by the disappearance of MWCNT characteristic peaks in their XRD patterns. In case of another study ${ }^{3}$ however. the XRD lines for the anatase type structure of MWNT-TiO 2 derived from MWCNT and $\mathrm{Ti}\left(\mathrm{OC}_{3} \mathrm{H}_{7}\right)_{4}$ at $673 \mathrm{~K}$ were observed the sharpened peaks and the separation of these diffraction peaks becomes clear. The XRD patterns of $\mathrm{TiO}_{2} /$ carbon composites by the study of MaldonadoHodar et al ${ }^{\hat{I}}{ }^{1)}$ showed the very similar results for the formation of anatase crystallites at low temperatures. The increase in the pyrolysis temperature. however. the pattems were shown the results in mixtures of anatase and rutile structures. This means that the increase in the heat treated temperature is include to phase transition from pure anatase phase to mixture phase of anatase and rutile or to rutile phase. In this study for the $\mathrm{CNT} / \mathrm{TiO}_{2}$ composite, the $\mathrm{TiO}_{2}$ structure shown was the anatase phase for the sample MCT. It was observed that the major peaks are diffractions from (101). (004). (200) and (204) planes of anatase. In case of sample HMCT, the main crystalline phase had presented the rutile plans of (101). (103). (112). (105), (211). (204). (116) and (220) with anatase plans. indicating the $\mathrm{TiO}_{2}$ prepared

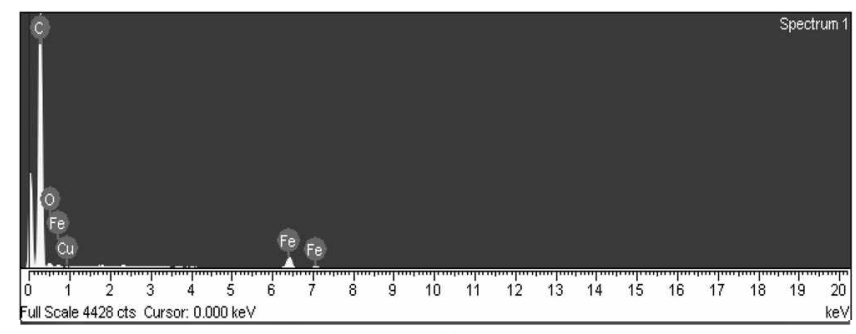

(a)

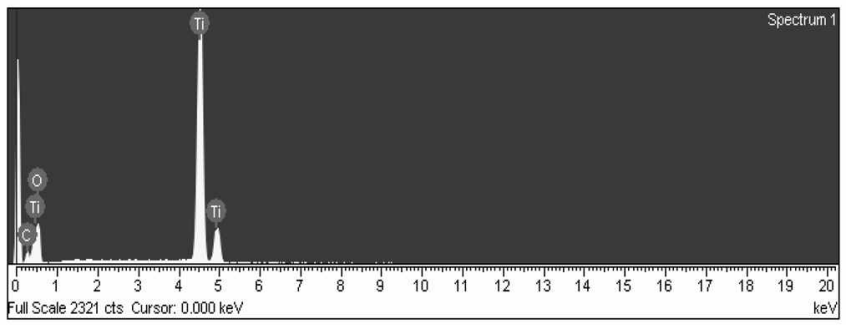

(b)

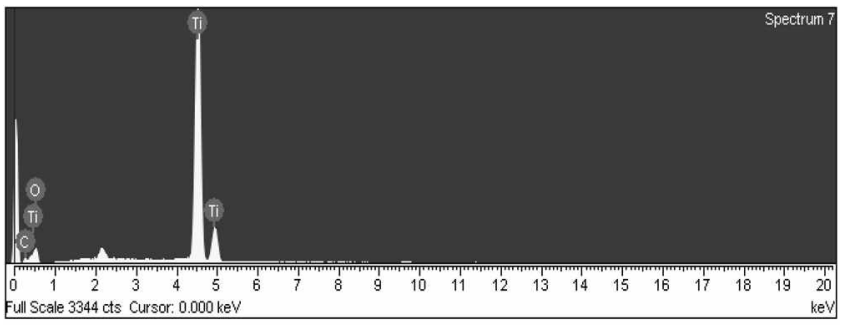

(c)

Figure 2. EDX elemental microanalysis for the $\mathrm{CNT} / \mathrm{TiO}$ s composites; (a) Pristine $\mathrm{TiO}_{2}$, (b) MCT composite catalyst and (c) HMCT composite catalyst.

existed in anatase and rutile mixture state.

The quantitative microanalysis of $\mathrm{C}$ and $\mathrm{Ti}$ as major elements for the $\mathrm{CNT} / \mathrm{TiO}_{2}$ composite was performed by EDX. The EDX spectra of CNT/TiO= composites were shown in Figure 2. The carbon coated solid particles and composite typed carbon for the elemental analyses has been reported on the various typed titania. ${ }^{21,22}$ In this study. the spectra were shown the presence as major elements of $C$ and $O$ with strong Ti peaks. However, it could be considered that the spectrum for the pristine MWCNT is shown the presence ad impurity element of $\mathrm{Fe}$. due to MWCNTs are synthesized by a catalytic chemical vapor deposition method in fluidized bed reactor on a Fe catalyst. The numerical result of EDX quantitative microanalysis of the $\mathrm{CNT} / \mathrm{TiO}_{2}$ composite was presented the 5.44:42,7.51.9 for the sample MCT and 3.09: 26.2:70.68 for the sample HMCT as components ratio of $C$ : Ti:O. In the case of most of the samples. oxygen and titanium were present as major elements with small quantities of carbon element in the $\mathrm{CNT} / \mathrm{TiO}_{2}$ composites. The results of EDX elemental microanalysis (wt.\%) of CNT/ $\mathrm{TiO}_{2}$ composites prepared with non and hydrochloric acid treatment to MWCNT listed in Table 2.

Surface morphology. The morphology and microstructure of the products prepared with or without acid were examined by SEM. The changes in the morphology for the MWCNT based composites obtained from our unique 
Table 2. EDX Elemental Microanalysis (We1ght \%) of $\mathrm{CNT} / \mathrm{TlO}_{2}$ Samples Prepared with Non and Hydrochloric Acid Treatment to MWCNT

\begin{tabular}{ccccc}
\hline Sample & $\mathrm{C}(\%)$ & $\mathrm{O}(\%)$ & $\mathrm{Ti}(\%)$ & Others (\%) \\
\hline Pristine CNT & 87.9 & 5.75 & - & 6.26 \\
MCT & 5.44 & 42.7 & 51.9 & - \\
HMCT & 3.09 & 26.2 & 70.68 & - \\
\hline
\end{tabular}

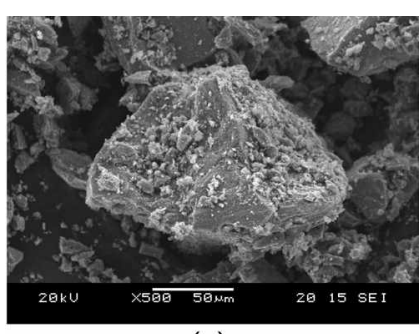

(a)

(c)

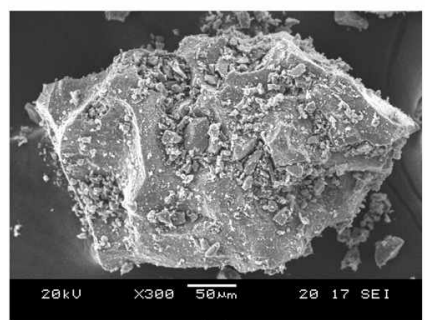

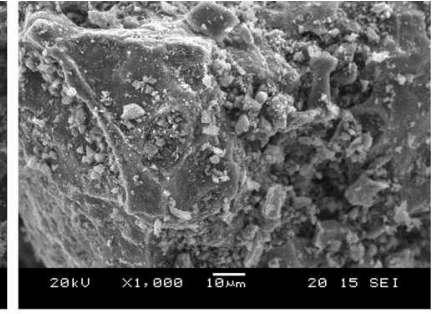

(b)

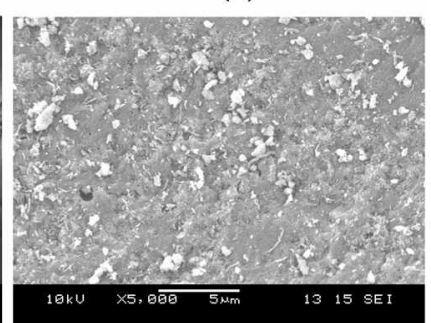

(d)
Figure 3. SEM micrographs for the CNT/TiO 2 composites; (a) MCT composite catalyst (over-all scale), (b) MCT composite catalyst (close-up), (c) HMCT composite catalyst (over-all scale) and (d) HMCT composite cataly st (close-up).

experimental condition were shown in Figure 3. In Figure 3. (a) and (b) showed a representative SEM images of the sample prepared without procedure of hydrochloric acid treatment (named as sample MCT), which clearly illustrates that the MWCNTs were homogenously decorated with welldispersed particles. It can be seen that the composites reveals the crystalline feature and well-resolved aligned lattice fringes of anatase. The low magnification images of the sample MCT (a) and sample HMCT (c) prepared by the thermal decomposition with TNB showed a homogenous sample with only individual MWCNTs covered with $\mathrm{TiO}_{2}$ without any jam-like aggregates between $\mathrm{CNTs}_{\text {and }} \mathrm{TiO}_{2}$. Higher magnifications (Fig. 3(b) and (d)) showed that all the MWCNTs are homogenously covered in the wall of $\mathrm{TiO}_{2}$ and $\mathrm{TiO}_{2}$ aggregates are not observed. These materials were showh microscopically compact and uniformly distribution and aggregates linked the MWCNTs resulting in a dense material. It was well explained that the good dispersion of small MWCNT particles into the $\mathrm{TiO}_{2}$ aggregates could provide the information on the existence of more reactive sites. due to a considerable portion of $\mathrm{TiO}_{2}$ would be enclosed in the three-dimensional matrix. Accordingly. a high photocatalytic yield was expected for a homogenous distribution of small MWCNT particles into the $\mathrm{TiO}_{2}$ aggregates. The higher photocatalytic activity of the $\mathrm{CNT} / \mathrm{TiO}_{2}$ composites night be attributed to the homogenous distri-
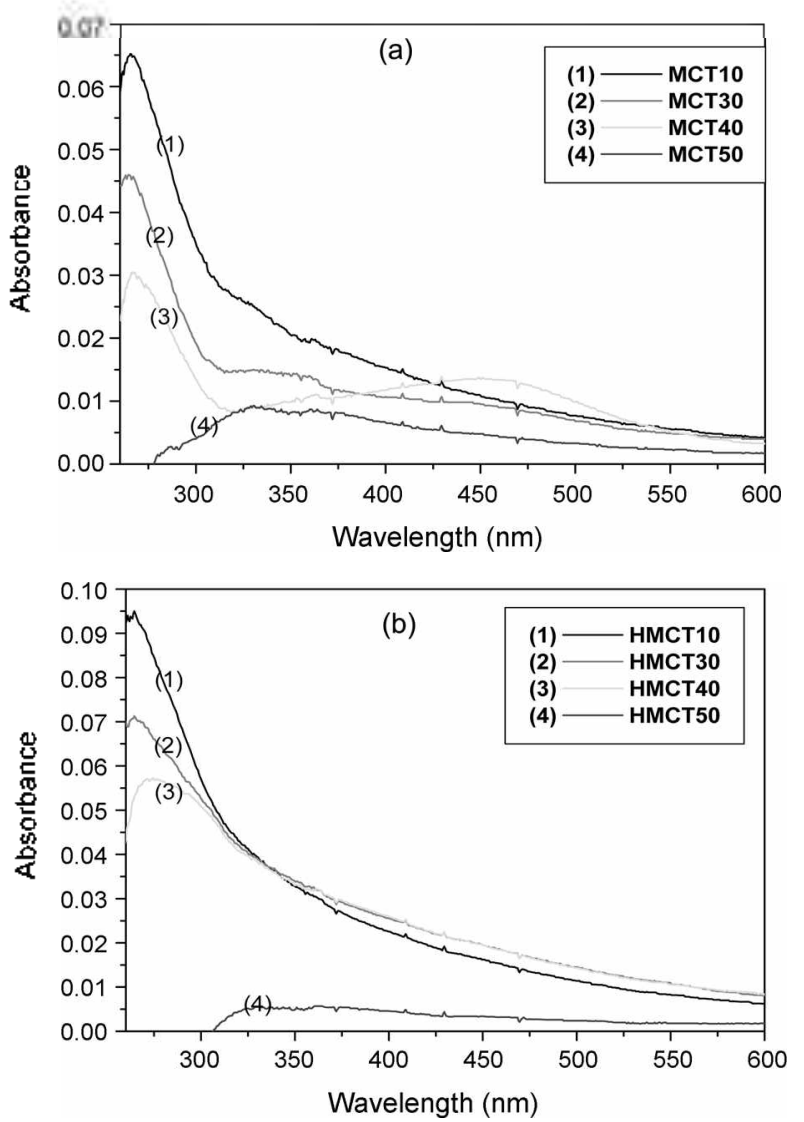

Figure 4. UV/VIS spectra of MB concentration against the CNT/ $\mathrm{TiO}_{2}$ composites under various irradiation time conditions; (a) MCT composite catalysts and (b) HMCT composite catalysts.

bution of CNT complexes and play important role as an energy sensitizer for improving the quantum efficiency and a charge transfer of the CNTs.

Photocatalytic effect of MB. Figure 4 showed changes by color disappearance in relative $\mathrm{MB}$ degradation concentration under UV light irradiation in the solution as a function of time. The $\mathrm{MB}$ degradation with $\mathrm{CNT} / \mathrm{TiO}_{2}$ composite was carried out to observe the UV photolysis effect. As shown in earlier study. ${ }^{11,14}$ if the MB concentration used is higher. " the intensity of absorbance maxima values could be estimate by photoproduct formed as a function of irradiation time. The fomation of absorbance maxima was proportional to the concentration decreased of the transient formed after the UV light excitation. However. if the concentration is lower. ${ }^{1+}$ the maxima values could not be certainly estimated. but absorbance at tried region decreased with an increase of degradation time. In this study. the measurement of absorbance from $220 \mathrm{~nm}$ to $600 \mathrm{~nm}$ region with MB products was tried as the function of photodegradation time by $\mathrm{CNT} / \mathrm{TiO}_{2}$ composites. It was shown that the maxima values were moved into long wavelength with a decrease to lower absorbance region with increasing irradiation time. It is considered that the light transparent of the MB solution highly increase by photocatalytic degradation effect.

The photocatalytic degradation of $\mathrm{MB}$ in aqueous solution containing CNT/TiO, composite photocatalyst under ultra- 


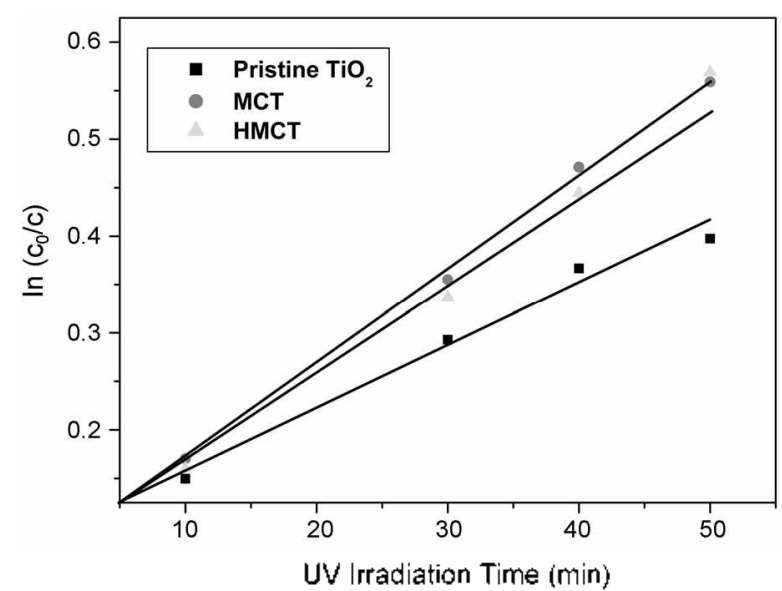

Figure 5. Apparent first-order linear transtom $\ln \left(c_{V} / c\right)$ against $f(t)$ of $\mathrm{MB}$ degradation kinetic plots for $\mathrm{TiO}_{2}, \mathrm{MCT}$ composite and HMCT composite

violet light irradiation follows pseudo-first-order kinetics. The kinetic plots were shown by apparent first-order linear transform $\ln (\mathrm{c} / \mathrm{c})$ against time function $(f(t))$ in Figure 5. The changes by color renoval were presented on the logarithm scale of relative concentration $\ln (\mathrm{c} / \mathrm{c})$ of $\mathrm{MB}$ degraded in the aqueous solution as a function of UV irradiation time. The appearance photodegradation effect might be due to the combination of the presence of UV light and an oxidation effect by dissolved oxygen in the suspension. The MB solution is quite unstable with variation of concentration when it was irradiated under UV light with $\mathrm{CNT} / \mathrm{TiO}_{2}$ composites. The relationship on the $\ln \left(\mathrm{c}_{\mathrm{i}} / \mathrm{c}\right)$ of $M B$ solution products as a time function under UV irradiation was shown linearity properties depending on kinetic first-order. As above mentioned. MWCNT had an energy sensitizer for improving the quantum efficiency with an increase of charge transfer. It is believed that the degradation of $\mathrm{MB}$ concentration in the aqueous solution can be occurred in two physical phenomena such as quantum efficiency by $\mathrm{CNT}$ and photocatalytic decomposition by $\mathrm{TiO}_{2}$. As a $\mathrm{MB}$ molecules absorbed energy from irradiation. therefore their delocalized electrons can be shift from bonding to antibonding orbital. Since MB adsorption likely occurs via $\pi \cdot \pi$ interactions between its delocalized electrons and the CNT's graphene layers, it is reasonable that shifts in its electron orbital would alter adsorption. The activity of the synthesized cataly'sts evaluated by comparing the apparent firstorder rate constants $\left(k_{\text {ippp }}\right)$ listed in Table 3. A combination factor $(\mathrm{R})$ was defined as $\mathrm{R}=k_{\text {apy }}\left(\mathrm{CNT} / \mathrm{TiO}_{2}\right) / k_{\text {app }}\left(\mathrm{TiO}_{2}\right)$ to quantify the combination effect. $\mathrm{TiO}_{2}$ and $\mathrm{CNT}_{2} \mathrm{TiO}_{2}$ composite photocatalyst (CT) give apparent rate constant of 6.8 $( \pm 0.1) \times 10^{-3}$ and $1.1( \pm 0.1) \times 10^{-2}$. respectively. The introduction of MWCNT into matrix obviously creates kinetic combination effect in MB degradation with an increase in the rate constant by the combination factor of 1.62 . In this paper the photocatalytic effect of $\mathrm{CNT} / \mathrm{TiO}_{2}$ composites could be attributed to the homogeneous aggregation of MWCNT in the $\mathrm{TiO}_{2}$ aggregates. The combination effect between MWCNT and $\mathrm{TiO}_{2}$ could be ascribed the enhanced
Table 3. The Apparent Kinetic Constant $\left(k_{\text {app }}\right)$ of $\mathrm{CNT} / \mathrm{TiO}_{2}$ Samples Prepared with Non and Hydrochloric Acid Treatment to MWCNT

\begin{tabular}{ccc}
\hline Sample & $k_{\text {app }}\left(\mathrm{min}^{-1}\right)$ & $\mathrm{R}$ \\
\hline Pristine $\mathrm{TiO}_{2}$ & $6.8( \pm 0.1) \times 10^{-3}$ & $\mathrm{~J}$ \\
$\mathrm{MCT}$ & $1.1( \pm 0.1) \times 10^{-2}$ & 1.62 \\
HMCT & $9.1( \pm 0.1) \times 10^{-3}$ & 1.33 \\
\hline
\end{tabular}

degradation of the $\mathrm{MB}$ as a pollutants on $\mathrm{CNT} / \mathrm{TiO}$. composite followed by a transfer through an interphase to titania where it is photo-degraded. According to earlier studies. ${ }^{21.24}$ the combination effects for the MB degradation should be attributed to the both effects between photocatalysis of the supported $\mathrm{TiO}_{2}$ and adsorptivity of the carbons. From the results of MB solution degraded during 50 minutes at $1.0 \times 10^{-6} \mathrm{~mol} / \mathrm{L}$ of initial MB concentration. it was shown that the kinetics for the $\mathrm{CNT} / \mathrm{TiO}_{2}$ composites results in a more significant degradation effect with increase of $\ln (\mathrm{c} / \mathrm{c})$ values than that of original $\mathrm{TiO}_{2}$. In the photocatalytic performance. it is suggested that the shade of $\mathrm{MB}$ concentration should be considered to the both effects between photocatalysis of the supported $\mathrm{TiO}_{2}$ and charge transfer on the MWCNT layers. The photocatalytic decomposition of pollutants occurs on $\mathrm{TiO}_{2}$ particles, even on those coated it on the carbon or aggregated it in the carbon. and that light absorption was actives on carbon particles. But, it is considered that carbon deep inside $\mathrm{TiO}_{2}$ is not easily

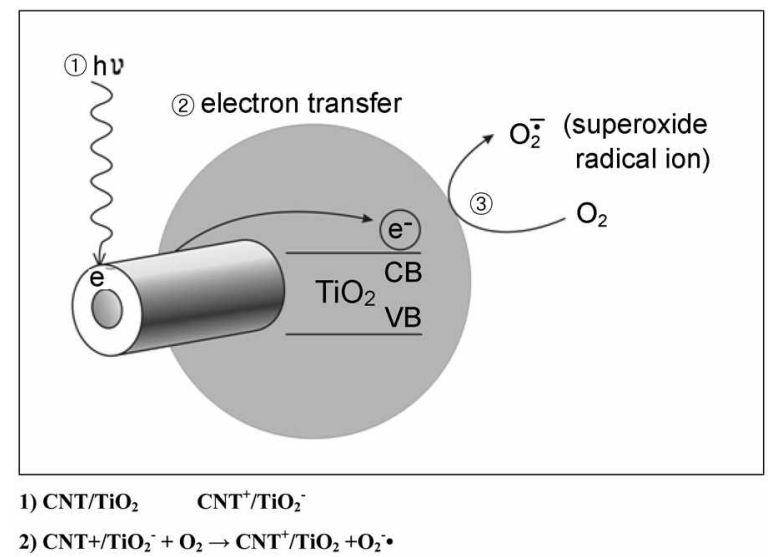

$\mathrm{CNT}^{+} / \mathrm{TiO}_{2}^{-}+\mathrm{O}_{2} \rightarrow \mathrm{CNT}^{+} / \mathrm{TiO}_{2}+\mathrm{O}_{2}^{-}$

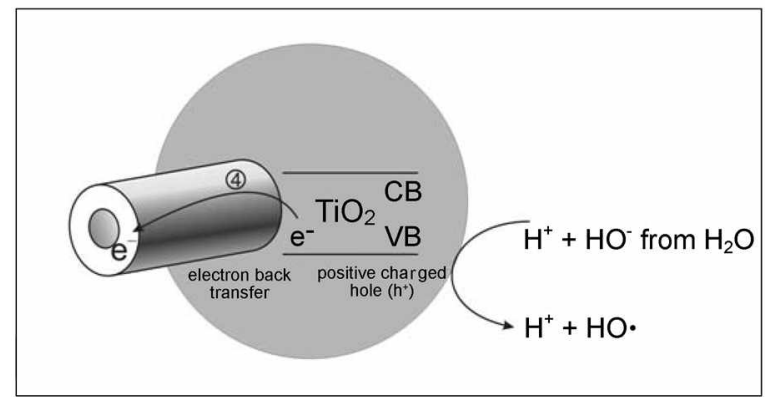

3) $\mathrm{CNT}^{+} / \mathrm{TiO}_{2} \rightarrow \mathrm{CNT} / \mathrm{TiO}_{2}{ }^{+}$

4) $\mathrm{CNT} / \mathrm{TiO}_{2}^{+}+\left(\mathrm{H}^{+}+\mathrm{OH}^{-}\right.$from $\left.\mathrm{H}_{2} \mathrm{O}\right) \rightarrow \mathrm{CNT} / \mathrm{TiO}_{2}+\mathrm{H}^{+}+\mathrm{HO}^{\cdot}$

Figure 6. Schematic diagram of mechanism to describe the combination effect to MWCNT acting as electron sensitizer and donator in the composite photocatalysts. 
accessible to light with excitation due to the long traveling distance and reflection and scattering by the support.

As plausible mechanism. it is quite reasonable to describe the combination effect to MWCNT acting as electron sensitizer and donator in the composite photocatalysts. The schematic diagram for the mechanism was shown in Figure 6. According to the semiconducting properties of CNT. MWCNT may accept the photo-induced electron $\left(\mathrm{e}^{-}\right)$by UV irradiation. It is considered that the electrons in MWCNT transfer into the conduction band in the $\mathrm{TiO}_{2}$ particles. In this time. this electrons in conduction band may react with $\mathrm{O}_{2}$. which can be trigger the formation of very reactive superoxide radical ion $\left(\mathrm{O}_{-}^{-}\right)$. Sinultaneously. a positive charged hole $\left(\mathrm{h}^{-}\right)$might be formed with electron transfer from valence bond in $\mathrm{TiO}_{2}$ to $\mathrm{MWCNT}$. The positive charged hole $\left(\mathrm{h}^{-}\right)$may react with the $\mathrm{OH}^{-}$derived from $\mathrm{H}_{2} \mathrm{O}$. which can be trigger the formation of hydroxyl radical (HO). Consequently, these radical groups are responsible for the decomposition of the organic compounds.

\section{Conclusions}

In this study. we present the synthesis and characterization of $\mathrm{CNT} / \mathrm{TiO}_{2}$ composites themally derived from MWCNTs and TNB by an improved oxidation method. The structural variations. elemental analysis and surface morphology are investigated through preparation of two kinds of $\mathrm{CNT} / \mathrm{TiO}_{2}$ composites. In the XRD patterns for the $\mathrm{CNT} / \mathrm{TiO}_{2}$ composites. the patterns reveal that only anatase phase can be identified for MCT composite. but the HMCT composite synthesized with $\mathrm{HCl}$ treatment was observed the mixed phase of anatase and rutile. The EDX spectra were shown the presence as major elements of $\mathrm{Ti}$ with strong peaks. From the SEM results the sample MCT and HMCT synthesized by the thermal decomposition with TNB were shown a homogenous sample with only individual MWCNTs covered with $\mathrm{TiO}_{2}$ without any jam-like aggregates between CNTs and $\mathrm{TiO}_{3}$. According to the photocatalytic results. the relationship on the $\ln (\mathrm{c} / \mathrm{c})$ of $\mathrm{MB}$ solution products as a time function under UV irradiation was shown linearity properties depending on kinetic first-order. From the results. it could be suggested the plausible photocatalytic mechanism between MWCNTs and $\mathrm{TiO}_{2}$.

\section{References}

1. Jitianu. A.: Cacciaguerra. T.: Benoit. R.: Delpeux. S.: Beguitr. F.: Bonnamy. S. Carbon 2004. 12. 1147-1151.

2. Sur1. I.: Iwasa. M.: Gao. L.: Zhang. Q. Carbon 2004. 885 901

3. Wang, W.: Serp, P; Kalck, P.: Faria. J. L. Jownal of Molecular Catalysis A: Chemical 2005, 235. 194-199.

4. Moreno-Castilla. C.: Maldonado-Hodar. T. F.: Carrasco-Marin. F.: Rodrigues-Castellon. E. Langzmmir 2012. 18. $2295-2299$.

5. Orlanducci. S.: Sessa. V.: Terranova. M. L.: Battiston. G. A.: Battiston, S: Gergasi, R. Carbon 2006. 41. $2839-2843$.

6. O'Connell, M. J. Carbon Nanotubes: Properties and Application' Tayor \& Francis: 2006: p 213.

7. Kaneko. M.: Oktura. I. Photocatalysis. Science and Techtologn: Kodansha \& Springer: 1999: p 124.

8. Ollis. D. F.: Alekabi. H. Photcatalytic Purification and Theatment of What and Air: Elsevier: 1993.

9. Dargon, G.; Tomkiewicz. M. J. Pln! Chem 1993, 97(49). 1265112655 .

10. Robert. D.: Parra. S.: Pulgarin. C.: Kızton. A.: Weber. J. V. Appl. Surf. Sci. 2000. 167. 51-58.

11. Oh. W. C.: Han. S. B.: Bae. T. S. Analyical Science \& Techology 2007. $20(4), 279-288$

12. Oh. W. C. Chen, M. L. Carbon Science 2007. 8(2), $108-$ 144.

13. Oh. W. C.: Chen. M. L.: Lim. C. S. Jonnal of Ceramic Processing Research 2007. 8(2). 119-124.

14. Oh. W. C.: Chen. M. L.: Ko. Y. S. Carbon Science 2007. 8(1). $6-$ 11.

15. Seeger. T: Redlish. P.: Grobert, N.; Terrones. M.; Walton. D. R. M. Kroto, H. W. Chem. Phus, Lett. 2001. 339. 41-46.

16. Hernadi. K.: Ljubbovic. E.: Seo. J. W.: Forro. L. Acta Mater. 2003. 5l. $1447-1+452$.

17. Cao. G. Namostructures \& Nanonaterials: Imperial College Press: 2004: p 3.4.

18. Yu. K.: Zhao, J.; Tian, Y.: Jiang. M.: Ding. X.; Liu, Y.: Zhu. Y; Wang, Z. Materials Letters 2005. 59, 3563-3566.

19. Oh. W. C.: Bae. T. S.: Chen. M. L. Corbon Science 2006. 7(4). 259-265.

20. Maldonado-Hodar. F. J.: Moreno-Castilla. C.: Rivera-Utrilla. J. Applied Catalysis A: General 2000, 203. 151-159.

21. Oh. W. C: Bae J. S.: Chen, M. L.; Ko, Y. S. Anatyical Science \& Techologu 2006, 19(5). 376-382.

22. Oh. W. C.: Bae. J. S.: Cher1. M. L. Bull. Korem Chem. Soc. 2006. 27(9). 1423-1328.

23. Oh. W. C.: Bae. J. S.: Chen. M. L. Analytical Science \& Techologu 2006, 19(6). $460-467$.

24. Inagaki, M.: Hirose, Y; Matsunage, T; Tsumura. T.: Toyoda M Carbon 2003. 41. 2619-2624. 